\title{
A ESPERANÇA EM DOENTES INTERNADOS EM UNIDADES DE CUIDADOS CONTINUADOS
}

\author{
| Rosa Martins¹; Micaela Domingues²; Ana Andrade³; Madalena Cunha4; Conceição Martins ${ }^{5}$ |
}

\section{RESUMO}

CONTEXTO: A vulnerabilidade da pessoa humana emerge quando a doença surge como uma ameaça, provocando desmoralização, desespero e sofrimento. O binómio saúde/doença parece ser afetado de forma positiva pela esperança, ajudando a pessoa/família a lidar com as incertezas do futuro de uma forma mais eficaz.

OBJETIVOS: Avaliar níveis de esperança em doentes internados em Unidades de Cuidados Continuados (UCCs), e identificar determinantes sociodemográficas, clínicas e psicossociais correlacionadas com esse constructo.

METODOLOGIA: Estudo quantitativo, transversal, descritivo e correlacional. Foi realizado em duas UCCs da região Centro de Portugal e o questionário foi aplicado a 92 doentes, com uma média de idade de 74,39 anos. O instrumento de recolha de dados integrou, variáveis sociodemográficas, clínicas, a escala de Esperança (Herth Hope Index), a escala de Qualidade de Vida (Functinal Assessement of Cancer Therapy) e o Questionário de Sono de Oviedo

RESULTADOS: Os dados mostram que 45,7\% dos inquiridos apresentam esperança reduzida, 39,1\% esperança elevada e 15,2\% esperança moderada. Apenas o "bem-estar funcional" da QDV e os "fenómenos adversos" da escala do sono se correlacionam significativamente ( $p=0,000 ; p=0,035)$ com a esperança explicando respetivamente $55,2 \%$ e $31,1 \%$ da sua variância. Já o género, idade, estado civil, situação profissional, escolaridade, rendimento mensal, tipologia e número de internamentos, mostraram não se correlacionar com a esperança.

CONCLUSÕES: A esperança é uma crença ou virtude inerente ao Homem, que assume níveis diferenciados, e acompanha o ser humano no seu processo de viver e de morrer condicionando ajustes nos momentos de crise, afetando e/ou sendo afetada pelo bem-estar e a qualidade de vida.

PALAVRAS-CHAVE: Esperança; Doentes; Cuidados Continuados; Qualidade de Vida

\section{RESUMEN}

\section{“A Esperanza en Doentes Internados en Unidades de Cuidados Continuos"}

CONTEXTO: Una vulnerabilidad da pessoa humana surge cuando una enfermedad surge como una amenaza, provocando desmoralización, desespero y sofrimento. O binómio saúde/ enfermedad parece estar afectado de forma positiva por la esperanza, ayudando a persona e familia a lidar com las incertezas de futuro de una forma más eficaz.

OBJETIVOS: Avaliar los niveles de esperanza en los internados en UCCs, identificando determinantes sociodemográficas, clínicas y psicossociales correlacionadas con este constructo.

METODOLOGÍA: Estudo cuantitativo, transversal, descritivo y correlacional. Se realizó en dos UCCs de la región Centro de Portugal y se aplicó un cuestionario a 92 pacientes, con una media de edad de 74,39 años. El instrumento de recogida de datos integrava, variables sociales y clínicas, una escala de esperanza, una escala de calidad de vida y el cuestionario de Sono de Oviedo.

RESULTADOS: Los datos muestran que $45,7 \%$ de los enfermos presentan esperanza reducida, $39,1 \%$ esperanza elevada y $15,2 \%$ esperanza moderada. Sólo el bienestar funcional de la QDV y los fenómenos adversos de la escala del sueño se correlacionam significativamente $(\mathrm{p}=0,000 ; \mathrm{p}=0,035)$ con la esperanza explicando respetivamente $55,2 \%$ y $31,1 \%$ de su variância. Ya el género, edad, estado civil, situación profesional, escolaridad, rendimiento mensual, tipología y número de internamentos, mostraron no se correlacionar con la esperanza.

CONCLUSIONES: La esperanza es una creencia o virtud inherente al Hombre, que asume niveles diferenciados, y acompaña el ser humano en su proceso de vivir y de morir condicionando ajustes en los momentos de crisis, afectando y/o siendo afectada por el bienestar y la calidad de vida.

\section{DESCRIPTORES: Esperanza; Enfermos; Cuidados Continu-} ados; Calidad de Vida

\begin{abstract}
"Hope in Hospitalized Patients on Continuous Care Units"

BACKGROUND: The vulnerability of the human person emerges when the disease appears as a threat, causing demoralization, despair and suffering. The binomial health / disease appears to be affected positively by hope, helping the person / family to deal with the uncertainties of the future more effectively.

AIM: To assess levels of hope in patients admitted to Continuous Care Units (CCU), and to identify sociodemographic, clinical, and psychosocial determinants correlated with this construct.

METHODS: Quantitative, transversal, descriptive and correlational study. It was performed in two CCU of the central region of Portugal and the questionnaire was applied to 92 patients, with a mean age of 74.39 years. The data collection instrument integrated, sociodemographic, clinical, Hope Scale (Herth Hope Index), the Quality of Life Scale (Functinal Assessement of Cancer Therapy) and Sleep Questionnaire Oviedo.

RESULTS: The data show that $45.7 \%$ of the respondents present less hope, $39.1 \%$ high hope and $15.2 \%$ moderate hope. Only the Quality of Life, "functional well-being" and the "adverse phenomena" of the sleep scale correlate significantly $(\mathrm{p}=0.000 ; \mathrm{p}=0.035)$ with the expectation explaining respectively $55.2 \%$ and $31.1 \%$ of their variance. The gender, age, marital status, professional status, schooling, monthly income, typology and number of hospitalizations showed no correlation with hope.

CONCLUSIONS: Hope is a belief or virtue inherent in man, which assumes different levels, and accompanies the human being in the process of living and dying conditioning adjustments in times of crisis, affecting and / or being affected by the well-being and quality of life.
\end{abstract}

\section{KEYWORDS: Hope, Patients, Continuing Care, Quality of Life}

Submetido em 31-01-2017

Aceite em 30-05-2017

1 Doutor; Professor Coordenador; Instituto Politécnico de Viseu, Unidade Cientifica-Pedagógica de Enfermagem de Reabilitação, Portugal, rmartins.viseu@gmail.com 2 Enfermeira Especialista em Enfermagem de Reabilitação; Unidade de Cuidados Continuados de Seia, Portugal, micas84@gmail.com

3 Doutor; Professor Adjunto; Instituto Politécnico de Viseu, Unidade Cientifica-Pedagógica de Enfermagem em Saúde Comunitária, Portugal, aandrade@essv.ipv.pt

4 Doutor; Professor Adjunto; Instituto Politécnico de Viseu, Unidade Cientifica-Pedagógica de Enfermagem de Medico Cirúrgica, Portugal, madalenacunhanunes@gmail.com 5 Doutor; Professor Coordenador; Instituto Politécnico de Viseu, Unidade Cientifica-Pedagógica de Enfermagem de Medico Cirúrgica, Portugal, mcamartinsp@gmail.com

Citação: Martins, R., Domingues, M., Andrade, A., Cunha, M., \& Martins, C. (2017). A esperança em doentes internados em unidades de cuidados continuados. Revista Portuguesa de Enfermagem de Saúde Mental (Spe. 5), 81-85.. 


\section{INTRODUÇÃO}

Atualmente as pessoas com doenças crónicas e de evolução prolongada são cada vez mais, uma realidade nos serviços de internamento dos Hospitais e das Unidades de Cuidados Continuados Integrados (UCCI). Os utentes que permanecem nestas unidades são geralmente pessoas que apresentam dependência funcional por falta ou perda de autonomia física, psíquica ou intelectual, resultante ou agravada por doença crónica, demência orgânica, sequelas pós -traumáticas, deficiência, doença severa e ou incurável em fase avançada (Querido, 2010). Estes quadros no seu conjunto são indutores de grande sofrimento, levando muitas vezes a pessoa a questionar-se sobre o sentido da vida, razão pela qual o "focus" da esperança nestes doentes assume uma importância fundamental.

$\mathrm{O}$ interesse pelo estudo da esperança tem estado associado aos doentes oncológicos, porém qualquer pessoa em situação de doença, mesmo que seja uma fase transitória se sente ameaçada e insegura. No caso dos doentes crónicos, estes sentimentos aumentam à medida que a doença evolui, com a instalação da debilidade física associada, incapacidade funcional, perda de papéis e consequentemente perda progressiva da esperança. A relevância e o reconhecimento do conceito de esperança tem vindo a crescer de forma significativa na Saúde e tem vindo a ter um impacto fulcral na prestação de cuidados de enfermagem (Gaspar, 2013). O conceito de esperança tem sido entendido não apenas como um sentimento individualizado e subjetivo, mas como uma experiência de sentido e propósito para a vida, que representa um componente essencial da prática de cuidados (Querido e Dixe, 2010).

$\mathrm{Na}$ verdade, e de acordo com os mesmos autores, a literatura tem vindo a realçar a importância da esperança nas pessoas doentes, descrevendo-a como força interior promotora de vida, facilitadora da transcendência da situação presente e transição para uma nova consciência e enriquecimento do ser. Inerente à vida humana, a esperança ou a falta dela, aparece associada às várias dimensões da nossa existência, na esfera pessoal, profissional, familiar e social. O seu grau de intensidade, e a importância que assume na hierarquia de prioridades, varia ao longo da vida. É nesta riqueza multimodal de dimensões, enfoques e determinações que o profissional de saúde poderá constituir-se num ponto de ancoragem na atribulada viagem e transformações constante da esperança perante as vicissitudes da doença (Barbosa, 2010).
Como oposto da esperança, mas também referida com alguma frequência na literatura, encontramos a desesperança. Carpenito (2011) define-a como um estado emocional, subjectivo em que a pessoa não é capaz de perspectivar ou encontrar alternativas para os seus problemas pessoais ou para alcançar um bem que deseja. Não acredita que possa ser ajudada por terceiros, não conseguindo deste modo concentrar-se nem mobilizar o esforço necessário para alcançar os objectivos definidos.

Para Parker-Oliver (2012), existem quatro estadios ou fases da esperança: esperança de cura; de tratamento; de prolongamento da vida e de uma morte serena. Este entendimento reforça a ideia de dinamismo e mudança constante de foco da esperança ao longo do tempo, frequentemente modificada, realinhada e redimensionada. Também Martins \& Mestre (2014), defendem que a esperança possui um potencial intencional para a terapêutica, apoiando e justificando a posição na expectativa de alguma melhoria, tornando-se, desta forma, fundamental à mobilização de forças para o restabelecimento do estado de saúde do doente, ao nível biopsicossocial, uma vez que este é um importante preditor da saúde e da Qualidade de Vida.

Pelas razões apontadas, entendemos que a promoção da esperança é uma tarefa desafiante e da máxima importância no cuidar, também nas UCCIs, uma vez que harmoniza no doente um sentido de bem-estar profundo. E neste sentido, Dias \& Ribeiro (2014) dizemnos, que o Enfermeiro é o profissional de saúde privilegiado pela proximidade de relação com o doente quer em meio hospitalar quer nas UCCI, e por isso deve estar alertado e sensibilizado para o despiste de sinais de desesperança. Também Pinto (2012), defende que o enfermeiro, poderá ser uma peça fundamental na promoção da esperança, ajudando a pessoa a melhorar as crenças que tem nas suas capacidades através de intervenções que se podem revelar muito úteis, nomeadamente: evitar esconder ou disfarçar a verdade, ajudar o doente a estabelecer objetivos realistas, apoiar a revisão de vida e valorizar as conquistas obtidas, envolver a pessoa ativamente nos cuidados e orientar a família na promoção de fatores encorajantes.

Perante a complexidade situacional envolvente dos doentes em UCCI, torna-se fundamental que os profissionais de saúde desenvolvam interesse e compreensão acerca da relação entre a esperança e o sentido de vida, uma vez que ambos proporcionam coragem e um estado de "imunidade" que se vai focalizar num futuro com legitimas possibilidades, ainda que nalguns casos breve. 


\section{METODOLOGIA}

Trata-se de um estudo quantitativo, transversal, descritivo e correlaciona, que utilizou uma amostra do tipo não probabilístico, por conveniência, constituída por 92 doentes internados em duas UCCs da região Centro de Portugal. Os critérios de seleção estabelecidos foram: estar internado em UCCI e ter capacidade cognitiva para auto-avaliação (de acordo com o Mini Mental State Examination). A colheita de dados decorreu entre Dezembro de 2015 e Março de 2016 e o instrumento de colheita de dados utilizado integrava questões sociodemográficas, clínicas e as escalas da Esperança (Herth Hope Index), a escala de Qualidade de Vida (Functinal Assessement of Cancer Therapy) e o Questionário de Sono de Oviedo. A Herth Hope Index-PT é um instrumento que avalia a esperança e é constituído por um total de 12 itens, organizados numa única dimensão, podendo a pontuação final variar entre 12 e 48 pontos, sendo que quanto maior for a pontuação obtida, maior o nível de esperança. (Viana, Dixe \& Barbosa, 2010).

Todos os procedimentos foram efetuados segundo uma rigorosa conduta ética, (com autorizações por parte dos Conselhos de Administração das Instituições e respetivas Comissões de Ética), garantindo-se o anonimato e confidencialidade dos dados recolhidos. O tratamento estatístico foi efectuado através do programa Statiscal Package Social Science versão 19.0 para o Windows e Word Microsoft, e foi processado utilizando estatística descritiva e estatística inferencial.

\section{RESULTADOS}

A amostra é constituída por 92 participantes, sendo $54(58,7 \%)$ do sexo feminino e $38(41,3 \%)$ do sexo masculino. A idade oscila entre um valor mínimo de 46 e um máximo de 92 anos, correspondendo-lhe uma média de 74,39 anos, um desvio padrão de 13,15 e um coeficiente de variação de $17,67 \%$. A média de idades para o sexo feminino $(=81,22)$ é superior à do sexo masculino ( $=64,68$ ), apresentando os dois grupos uma dispersão moderada em torno da média. $\mathrm{O}$ maior grupo percentual $(41,33 \%)$ da amostra é casada ou vive em união de facto, encontra-se em situação de reforma $(84,8 \%)$, reside em meio rural $(63,0 \%)$, apresenta baixa escolaridade (52,2\% ensino básico) e aufere de rendimentos mensais equivalentes ao ordenado mínimo nacional $(87,0 \%)$.
Em termos clínicos, verificámos que a maioria $(71,7 \%)$ dos doentes estão internados em Unidades de $\mathrm{Me}$ dia Duração e Reabilitação $(71,7 \%)$ e Unidades de Longa Duração e Manutenção (28,3\%), com outros internamentos anteriores $(89,1 \%)$. Os diagnósticos atuais com maior incidência são as fraturas $(30,4 \%)$, os AVCs $(17,4 \%)$, as depressões $(15,2 \%)$ e as doenças respiratórias $(10,9 \%)$ enquanto os motivos principais do internamento são a reabilitação funcional $(95,7 \%)$ e cognitiva (17,4\%). Os antecedentes pessoais mais relatados são a hipertensão arterial $(45,7 \%)$, a diabetes Mellitus (26,1\%), e o alcoolismo (15,2\%). A qualidade de vida global dos inquiridos é tendencialmente má, ( $<2$ pontos) e os valores menos positivos foram encontrados aos níveis do bem estar físico $(=1,64)$ emocional $(=1,78)$ funcional $(=1,62)$ e qualidade de vida total $(=1,84)$. Já o bem-estar social/familiar é positivo $(=2,25)$. Similarmente, a qualidade do sono dos doentes é divergente já que $45,7 \%$ tem sono com qualidade, $15,2 \%$ com qualidade moderada e $34,8 \%$ sem qualidade. A esperança dos nos nossos doentes, oscila entre um valor mínimo de 21 e um máximo de 45 pontos, (valores possíveis min. $=12$ e máx. $=48$ pontos) $c o m$ um valor médio de 33,39, um desvio padrão de 6,46 e um coeficiente de variação de 19,34\% (dispersão moderada em torno da média). A média da esperança para o sexo feminino $(=32,85)$ é ligeiramente inferior à do sexo masculino $(=34,15)$, porém as diferenças estatísticas não são significativas $(\mathrm{p}=0,060)$. Na constituição de grupos com base na fórmula (Média \pm 0.25 dp), verificámos, que $45,7 \%$ dos doentes apresenta esperança reduzida, $39,1 \%$ esperança elevada e $15,2 \%$ esperança moderada (cf. tabela 1)

Tabela 1 - Níveis de esperança dos Participantes em função do sexo

\begin{tabular}{|c|c|c|}
\hline \multirow{2}{*}{ NIVEIS DE ESPERANÇA } & \multicolumn{2}{|c|}{ TOTAL } \\
\cline { 2 - 3 } & $\mathrm{N}$ & $\%$ \\
\hline Esperança reduzida & 42 & 45,7 \\
\hline Esperança moderada & 14 & 15,2 \\
\hline Esperança elevada & 36 & 39,1 \\
\hline
\end{tabular}

$\mathrm{Na}$ associação entre variáveis constatámos que apenas as dimensões "bem-estar funcional" da escala de QDV e os "fenómenos adversos" da escala do sono se correlacionam significativamente $(\mathrm{p}=0,000 ; \mathrm{p}=0,035)$ com a esperança explicando respetivamente $55,2 \%$ e $31,1 \%$ da sua variância. Já o género, idade, estado civil, situação profissional, escolaridade, rendimento mensal, tipologia e número de internamentos, mostraram não se correlacionar de forma significativa $(p>0,05)$ com a esperança destes doentes. 


\section{DISCUSSÃO}

Os resultados deste estudo mostram que a esperança é de facto um sentimento individualizado, subjetivo e experienciado de forma diferente de pessoa para pessoa uma vez que $45,7 \%$ dos doentes apresentam esperança reduzida, 39,1\% esperança elevada e $15,2 \%$ esperança moderada. Estes dados diferem dos de Martins \& Mestre (2014) ao afirmarem que $52 \%$ dos idosos investigados, apresentavam níveis elevados de esperança, contudo corroboram Querido (2005), ao defender a existência de um processo de manutenção da esperança ao longo da vivência da situação de doença, em que a capacidade humana para manter a esperança face à adversidade é na verdade considerável.A compreensão do contexto sociodemográfico e clinico dos doentes torna-se fundamental na análise da esperança se tivermos em conta que esta constitui uma fonte de energia que engloba aspetos físicos e psicossociais de busca de sentido.

A amostra é maioritariamente feminina (58,7\%), com uma média de idade de 74,39 anos, baixa escolaridade (52,2\% ensino básico) reformada, a residir em meio rural $(63,0 \%)$ e com baixos rendimentos. São dados que correspondem aos caracterizadores do Relatório de Monitorização da RNCCI (2015), em que o perfil de utentes da rede tem evidenciado uma população envelhecida, maioritariamente feminina, com baixo nível de escolaridade e carenciada. Em termos clínicos, existe um perfil de doentes com história de mais de um internamento $(89,1 \%)$, com diagnósticos principais de fraturas (30,4\%), AVCs $(17,4 \%)$, depressões $(15,2 \%)$ e doenças respiratórias (10,9\%). Apresentavam antecedentes pessoais de hipertensão arterial (45,7\%), diabetes Mellitus (26,1\%), e alcoolismo (15,2\%). Trata-se portanto de pessoas com quadros clínicos de patologias crónicas incuráveis e de evolução progressiva, em que muitos aspectos da vida são afectados e inter-relacionados acarretando ao doente e família um determinado tipo de sofrimento que põe em causa as suas conceções de esperança (Gaspar, 2013).

Pudemos ainda constatar, que os motivos principais do internamento nas UCCs são essencialmente a reabilitação funcional $(95,7 \%)$ e cognitiva $(17,4 \%)$ correspondendo estas aspirações às finalidades da RNCCI que visam prestar cuidados de saúde, centrados na pessoa e adaptados às suas necessidades, promovendo a restauração e manutenção do bem-estar, conforto e qualidade de vida da pessoa (Petronilho, 2012).
Os doentes apresentam má QDV em todas as dimensões, excepto no bem estar social e familiar que é positivo $(=2,25)$. Também estes dados corroboram os de Oliveira (2014) quando afirma que a perda de bemestar se traduzem em fenómenos únicos e subjetivos e o doente em sofrimento necessita da atenção de outras pessoas, sendo os amigos e família as principais fontes de o apoio. A dimensão "bem-estar funcional" da QDV correlaciona-se com a esperança de forma altamente significativa $(\mathrm{p}=0,000)$ explicando $55,2 \%$ da sua variância. Este dado mostra a importância das UCCs na reabilitação do doente, uma vez que a melhoria do seu estado funcional pode constituir só por si possíveis caminhos de esperança (Martins \& Mestre, 2014).

Por fim a qualidade do sono dos nossos doentes é diversificada, já que $45,7 \%$ tem sono com qualidade, $15,2 \%$ com qualidade moderada e $34,8 \%$ sem qualidade. São vários os estudos epidemiológicos sobre a população adulta em geral que indicam uma prevalência de má qualidade de sono entre os $15 \%$ e os $35 \%$, contudo sabemos que dormir bem é fundamental, pois a quantidade e a qualidade do sono são importantíssimos no processo de recuperação do doente (Silva, 2015). A dimensão "fenómenos adversos" da escala do sono correlaciona-se significativamente $(\mathrm{p}=0,035)$ com a esperança explicando $31,1 \%$ da sua variância. Não é ainda clara a relação entre a qualidade do sono qualidade de vida e a esperança, contudo a negatividade duas primeiras dimensões traduzem-se inevitavelmente em sofrimento, que por vezes leva o doente à desesperança.

\section{CONCLUSÃO}

As principais conclusões deste estudo vêm robustecer o paradigma de que os doentes internados nas UCCs são portadores de doenças crónicas, com evolução prolongada onde a doença leva á perda de bem-estar e ao sofrimento. Este não se reduz ao domínio físico mas ultrapassa-o nos seus domínios afetivos, cognitivos e espirituais, levando a Pessoa ao questionamento existencial e a procurar sentido para a vida. Percebemos que a esperança se traduz num sentimento individualizado e subjetivo, é vivida de forma única e pessoal, distribuindo-se num continuum que vai desde a esperança reduzida $(47,7 \%)$ esperança moderada $(15,2 \%)$ á esperança elevada $(39,1 \%)$. De destacar também o contexto que rodeia cada doente, nomeadamente o contexto sociodemográfico, clinico e psicossocial, sendo estes também sustentados na consciência que os próprios doentes tem de si próprios. 
De facto as condições sociodemográficas (idade avançada, baixa escolaridade e parcos recursos económi$\cos )$, clinicas (incapacidades físicas e emocionais), psicossociais (má QDV e perturbações do sono) que caracterizam estes doentes, não são facilitadoras da busca de um sentido para a vida, levando-os por vezes a formas de esperança perdida que é a desesperança.

\section{IMPLICAÇÕES PARA A PRÁTICA CLÍNICA}

Estas constatações levam-me a reforçar sugestões já apresentadas por outros autores, sobre a importância fulcral e o papel determinante dos profissionais de saúde em especial os enfermeiros na abordagem da problemática da esperança no contexto do doente internado em UCCs. Não temos dúvidas de que os enfermeiros estão numa posição privilegiada podendo influenciar os sentimentos de esperança de quem cuidam. Porém e para que isso seja possível, o enfermeiro terá de estar desperto e saber auscultar o significado que o doente atribui às ameaças, aos esforços adaptativos que abraça, aos contextos (pessoais, sociais e clínicos) promotores da esperança onde as suas intervenções especializadas podem atuar, no sentido do doente se adaptar a uma vida possivelmente reconstruída e modificada.

\section{REFERÊNCIAS BIBLIOGRÁFICAS}

Barbosa, A. (2010). A espiritualidade. In A. Barbosa, \& I. G. Neto (Coords), Manual de cuidados paliativos (2a ed., pp. 595-659). Lisboa: Faculdade de Medicina de Lisboa, Centro de Bioética.

Carpenito, L. J. (2011). Manual de diagnósticos de enfermagem (13 ${ }^{\mathrm{a}}$ ed.). Porto Alegre: Artmed Editora.

Dias, E. M., \& Ribeiro, J. P. (2014). Intervenção psicológica positiva em grupo: forças e virtudes na reabilitação pós-avc. Psicologia, Saúde \& Doenças, 15(1), 201- 218. doi.org/10.15309/14psd150117

Gaspar, S. M. C. (2013). Construindo laços de esperança num cuidar especializado ao doente em situação crítica. Dissertação de Mestrado, Universidade Católica Portuguesa, Lisboa Portugal. Acedido em http://repositorio.ucp.pt/handle/10400.14/14785

Martins, R. M. L., \& Mestre, M. A. (2014). Esperança e qualidade de vida em idosos. Millenium, 47, 153-162.
Oliveira, J. G. (2014). Terapia de esperança: Uma intervenção grupal que visa promover a esperança de idosos institucionalizados. Dissertação de Mestrado. Universidade de Coimbra, Coimbra, Portugal. Acedido em https://estudogeral.sib.uc.pt/handle/10316/15341

Parker-Oliver, D. (2012). Redefining hope for the terminally ill. American Journal of Hospice \& Palliative Care, 19(2), 115-120. doi: 10.1177/104990910201900210

Petronilho, F. A. S. (2012). Autocuidado: Conceito central da enfermagem. Coimbra: Formasau.

Pinto, S., Caldeira, S., \& Martins, J., C. (2012). A esperança da pessoa com cancro- estudo em contexto de quimioterapia. Revista de Enfermagem Referência, 3(7), 23-31.

Portugal, Ministério da Saúde. (2015). Relatório de monotorização do desenvolvimento e da atividade da Rede Nacional de Cuidados Continuados Integrados (RNCCI) - $1^{\circ}$ semestre de 2015. Lisboa: SNS. Acedido em http://www.acss.min-saude.pt/wp-content/ uploads/2016/07/Relatorio-de-Monitorizacao-Anualda-RNCCI-2014.pdf

Querido, A. I. F. (2005). Esperança em cuidados paliativos. Dissertação de Mestrado, Universidade de Lisboa, Lisboa, Portugal. Acedido em https://iconline. ipleiria.pt/handle/10400.8/120

Querido, A. I., \& Dixe, M. A. (2010). A esperança e qualidade de vida dos doentes em cuidados paliativos. International Journal of Developmental and Educational Psychology, 1(1) 613-622. Acedido em http:// infad.eu/RevistaINFAD/2010/n1/volumen $1 /$ IN FAD_010122_613-622.pdf

Silva, M. F. (2015). Determinantes da qualidade do sono em estudantes de enfermagem. Dissertação de Mestrado, Instituto Politécnico de Viseu, Escola de Saúde, Viseu, Portugal. Acedido em http://repositorio. ipv.pt/handle/10400.19/2865

Viana, A., Querido, A., Dixe, \& M. Barbosa, A. (2010) Avaliação da esperança em cuidados paliativos: Tradução e adaptação transcultural do Herth Hope Index. International Journal of Developmental and Educational Psychology, 2(2), 607-616. Acedido em http://infad.eu/RevistaINFAD/2010/n1/volumen2/ INFAD_010222_125-134.pdf 
